\title{
Planetary Nebulae as Tracers of the Intergalactic Stellar Background: a Population Synthesis Theoretical Approach
}

\author{
Alberto Buzzoni ${ }^{1}$ and Magda Arnaboldi ${ }^{2}$ \\ 1 INAF - Osservatorio Astronomico di Bologna, Via Ranzani 1, 40127 Bologna (Italy) \\ 2 INAF - Osservatorio Astronomico di Torino, Via Osservatorio 20, 10025 Pino \\ Torinese (Italy)
}

\begin{abstract}
We wish to assess the relationship between the population of planetary nebulae (PNe) and a given parent stellar population from a theoretical point of view. Our results rely on original population synthesis models used to estimate the expected luminosity-specific PN density accounting for different evolutionary scenarios and star formation histories, as observed in galaxies in the near Universe. For a complete PN sample, we find that $1 \mathrm{PN} / 1.510^{6} \mathrm{~L} \odot$ is a safe (IMF-independent) lower limit to the traced global bolometric luminosity of the parent stellar population. A tentative application to Virgo cluster data allows us to place a lower limit at $\sim 7 \%$ for the global B luminosity of the cluster provided by "loose" intergalactic stars.
\end{abstract}

\section{Introduction}

Recent works on diffuse light in nearby clusters have renewed interest in planetary nebulae $(\mathrm{PNe})$ as tracers of the parent stellar population. The efficient detection of these nebulae in the field, even at $70 \mathrm{kpc}$ or more beyond the optical radius of bright galaxies (see e.g. Peng et. al 2004), makes them ideal candidates to trace the "loose" stellar population possibly filling the "empty" space among galaxies. PNe can be detected up to in nearby clusters at a distance of $20 \mathrm{Mpc}$, like for instance for the Virgo or the Fornax cluster (see Arnaboldi et al. 2003; Feldmeier et al. 2003).

A major issue in this regard concerns the link between PN number density and global (bolometric) luminosity of their parent stellar population. This ratio (the so-called "luminosity-specific PN density" or, shortly, the " $\alpha$ ratio", as defined first by Jacoby 1980) provides in fact direct information on the distinctive physical properties of the underlying stellar population as far as its PN component can be picked up by the observations.

Population synthesis models can be especially useful in this sense, allowing us to tackle the problem from a fully theoretical point of view, thus overcoming most of the natural limits of the empirical calibrations (i.e. observational bias, incomplete sampling etc.). On this line, we want to give here a brief progress report of our project aimed at calibrating " $\alpha$ " for different evolutionary scenarios and star formation histories as observed in the local galaxy sample and likely consistent with the expected properties of the diffuse stellar component of the intergalactic medium. 


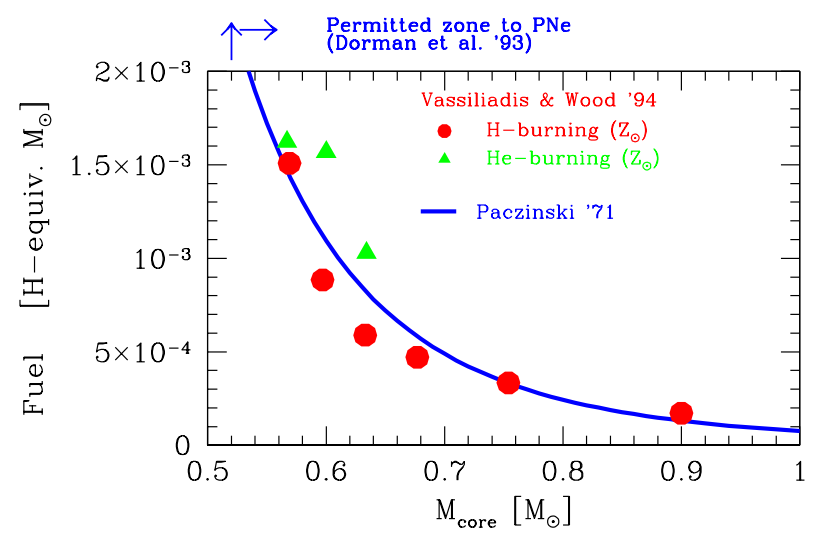

Fig. 1. Theoretical fuel consumption for stars along the Hot-PAGB evolution according to the models of Paczynski (1971; solid curve) and Vassiliadis and Wood (1994; solid dots and triangles). Fuel is expressed in Hydrogen-equivalent solar mass. The stellar core-mass range to produce PNe is marked, after Dorman et al. (1993).

\section{Theoretical fundamentals}

Theory of simple stellar populations (SSPs), as developed in Renzini \& Buzzoni (1986) and Buzzoni (1989) (hereafter RB86 and B89, respectively), provides the basic reference framework for our task, allowing us to evaluate the value of " $\alpha$ " for coeval and chemically homogenous stellar aggregates.

Briefly, the expected number $\left(N_{\mathrm{PN}}\right)$ of PNe for a SSP of total (bolometric) luminosity $L_{t o t}$ is:

$$
N_{\mathrm{PN}}=\mathcal{B} L_{\mathrm{tot}} \tau_{\mathrm{PN}}
$$

In the equation, $\mathcal{B}$ is the so-called "specific evolutionary flux" (a nearly age- and IMF-independent parameter of the order of $210^{-11}\left[L_{\odot}^{-1} \mathrm{yr}^{-1}\right]$, see B89), and $\tau_{\mathrm{PN}}$ is the PN lifetime (i.e. the time for the nebula to be detectable in [O III] and/or $\mathrm{H} \alpha$ surveys).

The PN lifetime directly modulates with the stellar core-mass evolution, the latter depending on the nuclear fuel burnt at high temperature $\left(T_{\text {eff }} \simeq 10^{5} \mathrm{~K}\right)$ along the Hot Post-AGB (H-PAGB) phase (this is a direct result of the socalled "Fuel Consumption Theorem" of RB86). Figure 1 summarizes the model predictions, after Paczynski (1971) and Vassiliadis and Wood (1994), displaying the H-PAGB fuel consumption for stars of different core masses. ${ }^{1}$ In the plot, we express the fuel in terms of Hydrogen-equivalent solar mass (i.e. $1 \mathrm{~g}$ of $\mathrm{H}$ equivalent mass $=610^{18}$ ergs; cf. RB86).

The inferred H-PAGB lifetime for SSPs of different age, according to B89, is reported in Fig. 2 . It can be defined as $\tau_{\mathrm{HPAGB}} \simeq$ Fuel $/ \ell_{\mathrm{H}-\mathrm{PAGB}}$, being $\ell_{\mathrm{H}-\mathrm{PAGB}}$

\footnotetext{
${ }^{1}$ Note that actual PAGB core mass of stars can sensibly be lower than the original stellar mass when leaving the Main Sequence Turn Off point. This is a consequence of the mass loss processes that heavily affect stellar evolution along the red and asymptotic giant branch phases.
} 


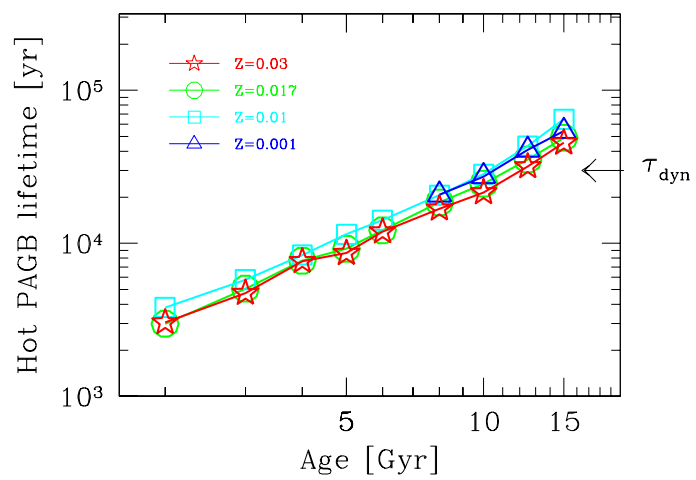

Fig. 2. Hot-PAGB core mass lifetime, for SSP evolution according to B89 synthesis models. A Salpeter IMF is assumed, and a Reimers mass loss rate coefficient $\eta=0.3$. The explored metallicity spans the range from $Z \sim 1 / 20 \rightarrow 2 Z_{\odot}$, as labeled in the plot. The PN dynamical timescale $\left(\tau_{\mathrm{dyn}}\right)$ is also marked, for reference.

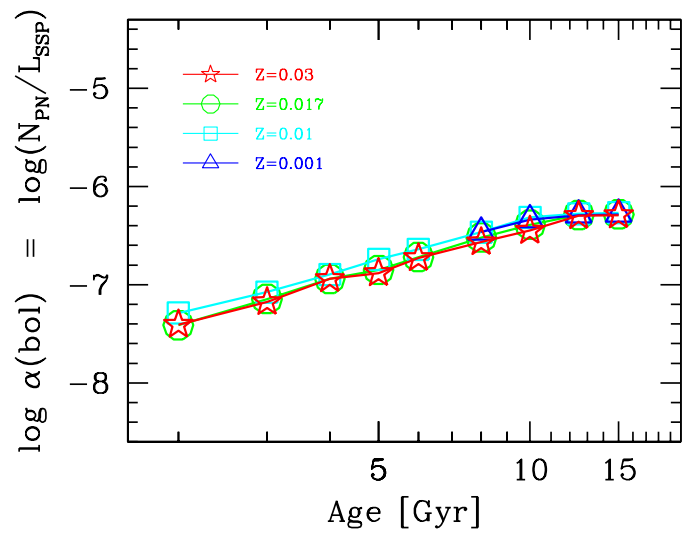

Fig. 3. Theoretical luminosity-specific PN density for SSPs of different metallicity. Models are according to B89, with the distinctive parameters as in Fig. 2.

the luminosity of the stellar core at the onset of the nebula ejection (see e.g. Paczynski 1971 for details). It is important to note that, in general, $\tau_{\text {HPAGB }}$ is shorter than the dynamical timescale for nebula evaporation $\left(\tau_{\mathrm{dyn}} \simeq 30000 \mathrm{yr}\right.$; cf. Peimbert 1990); this implies that, at least for SSPs of young and intermediate age, $\tau_{\mathrm{PN}}$ should actually coincide with $\tau_{\mathrm{HPAGB}}$. Only for $t_{\mathrm{SSP}} \gtrsim 12$ Gyr we have that $\tau_{\mathrm{PN}} \simeq \tau_{\mathrm{dyn}}$, so that the luminosity-specific PN density could eventually be written as

$$
\alpha=\frac{N_{\mathrm{PN}}}{L_{\mathrm{SSP}}}=\mathcal{B} \tau_{\mathrm{PN}}=\mathcal{B} \inf \left\{\tau_{\mathrm{HPAGB}}, \tau_{\mathrm{dyn}}\right\}
$$

From eq. (2), and taking the B89 synthesis code as a theoretical reference, in Fig. 3 we report the expected luminosity-specific PN density for SSPs of different metallicity. Some physical age constraints exist for SSPs to produce 


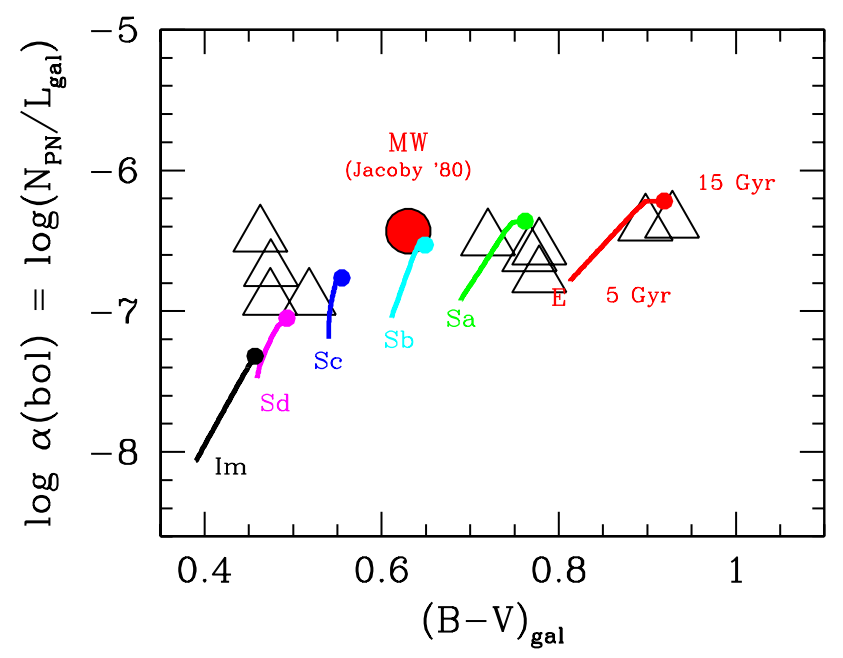

Fig. 4. Expected luminosity-specific PN density for template galaxy models (labeled with the corresponding Hubble type), according to Buzzoni (2002). Each curve tracks galaxy evolution from 5 Gyr to 15 Gyr (small solid dots), as labeled for the case of Ellipticals. Big triangles report the empirical estimate of $\alpha=N_{\mathrm{PN}} / L_{\text {gal }}$ from the PN census of Jacoby (1980) for the galaxies in the Local Group. The case of the Milky Way is singled out in the plot by the big solid dot.

$\mathrm{PNe}$; from one hand, for $t_{\mathrm{SSP}} \lesssim 10^{8} \mathrm{yr}$ SSP evolution is dominated by stars with $\mathrm{M}_{*} \gtrsim 5 \mathrm{M}_{\odot}$ ending up as Supernovae. On the other hand, no PNe are expected to form from stars with actual mass $\mathrm{M}_{*} \lesssim 0.52 \mathrm{M}_{\odot}$ (Dorman et al. 1993; see Fig. 1); this is likely to occur at some point along SSP evolution for ages between $10 \lesssim t_{\mathrm{SSP}} \lesssim 18 \mathrm{Gyr}$, the exact details depending on the efficiency of the mass loss processes ${ }^{2}$.

\section{The luminosity-specific PN density in the galaxies: implications for the intergalactic medium}

The theoretical estimate of the luminosity-specific PN density is important when we infer total bolometric luminosity of their parent stellar population in the intergalactic medium. So far, we have relied upon the empirical calibration of $\alpha$ on the bulge of M31, with all the uncertainties induced by the poorly understood effects of metallicity, age and star formation history.

Our new results, based on this improved theoretical approach, indicate that a luminosity-specific PN density

$$
\alpha=1 \mathrm{PN} / 1.510^{6} \mathrm{~L}_{\odot}
$$

\footnotetext{
${ }^{2}$ The B89 SSP models parameterize mass loss via the coefficient $\eta$ according to the Reimers (1975) standard notation. A value of $\eta=0.3$ is adopted here, as an indicative value for the observed mass loss in the Galactic globular clusters (see B89 for details).
} 
is a safe estimate for old SSPs. Quite interestingly, this value does not strongly depend on metallicity and, even more importantly, on the stellar IMF (as $\mathcal{B}$, in eq. (2), is nearly insensitive to the IMF details, as shown by RB86).

On the basis of the derived SSP evolution, and matching the Buzzoni (2002) template galaxy models, we have further elaborated our synthesis scheme computing in Fig. 4 the expected evolution of $\alpha$ for the different star formation histories of galaxies along the Hubble morphological sequence. A comparison with the observed PN population for the Local Group members (Jacoby 1980, including the relevant case of the Milky Way) is also reported in the figure.

As a tentative application of our results, we could match the recent observations of the Virgo cluster by Arnaboldi et al. (2003) in order to assess the luminosity contribution from the intergalactic stellar background. Some 37 PN candidates have been detected by Arnaboldi et al. ( $\sim 25 \%$ of which possibly consisting of spurious $\operatorname{Ly} \alpha$ emitters at $z=3.14$, as claimed by the authors) across an area of $0.196 \mathrm{deg}^{2}$ on the cluster. As the bona fide PNe only span the brightest magnitude bin of the standard luminosity function we had to correct for sample incompleteness according to Ciardullo et al. (1989).

By adopting the reference range of $\alpha$ for 15 Gyr galaxy models of Fig. 4, an intergalactic surface brightness (in bolometric) could be derived as $26.0 \lesssim \mu_{\text {bol }} \lesssim$ $28.5 \mathrm{mag} \operatorname{arcsec}^{-2}$, depending on the assumed star formation history for the PN parent stellar population. ${ }^{3}$ This implies a B surface brightness for intergalactic stars in the range $27.3 \lesssim \mu_{\mathrm{B}} \lesssim 30.3$, mag $\operatorname{arcsec}^{-2}$. Compared to the smooth B surface brightness distribution of the bright-galaxy component of Virgo (i.e. $\mu_{\mathrm{B}} \simeq 27.4 \mathrm{mag} \operatorname{arcsec}^{-2}$, see Arnaboldi et al. 2003), this allows us to place a confident lower limit to $7 \%$ for the cluster global luminosity to be provided by "loose" intergalactic stars.

\section{References}

1. M. Arnaboldi et al.: Astron. Journal 125, 514 (2003)

2. A. Buzzoni: Astrophys. Journal Suppl. Ser. 71, 817 (1989) [B89]

3. A. Buzzoni: Astron. Journal 123, 1188 (2002)

4. R. Ciardullo, G. H. Jacoby, H. C. Ford, J. D. Neill: Astrophys. Journal 339, 53

5. B. Dorman, R. T. Rood, R. W. O'Connell: Astrophys. Journal 419, 596 (1993)

6. J. J. Feldmeier, R. Ciardullo, G. H. Jacoby, P. R. Durrell: Astrophys. Journal Suppl. Ser. 145, 65 (2003)

7. G. Jacoby: ApJ 42, 1 (1980)

8. B. Paczynski: Acta Astr. 21, 47 (1971)

9. M. Peimbert: Rev. Mexicana Astron. Astrof. 20, 119 (1990)

10. E. W. Peng, H. C. Ford, K. C. Freeman: Astrophys. Journal 602, 685 (2004)

11. D. Reimers: Mem. Soc. Roy. Sci. Liège, 6th Ser. 8, 87 (1975)

12. A. Renzini A. Buzzoni: 'Global properties of stellar populations and spectral evolution of galaxies'. In Spectral Evolution of Galaxies, ed. C. Chiosi, A. Renzini (Reidel, Dordrecht 1986) pp. 195-235 [RB86]

13. E. Vassiliadis, P. R. Wood: Astrophys. Journal Suppl. Ser. 92, 125 (1994)

\footnotetext{
${ }^{3}$ For our calculations we adopt a distance modulus $(m-M)=30.8$ mag for the Virgo cluster, and an absolute bolometric magnitude $M_{\mathrm{bol}, \odot}=4.72$ for the Sun.
} 